\title{
Validità delle teorie idrodinamiche sulle sesse: conferme dei laghi di Levico e Caldonazzo (*)
}

\section{Pietro Caloi}

\author{
Ricevuto il 31 Luglio 1961
}

In lavori precedenti $(1,2,3)$, sono state calcolate, per via teorica, le caratteristiche fondamentali delle oscillazioni libere dei laghi di Levico e Caldonazzo. Furono applicati tre diversi metodi per il lago di Levico e due per quello di Caldonazzo.

Tabella I

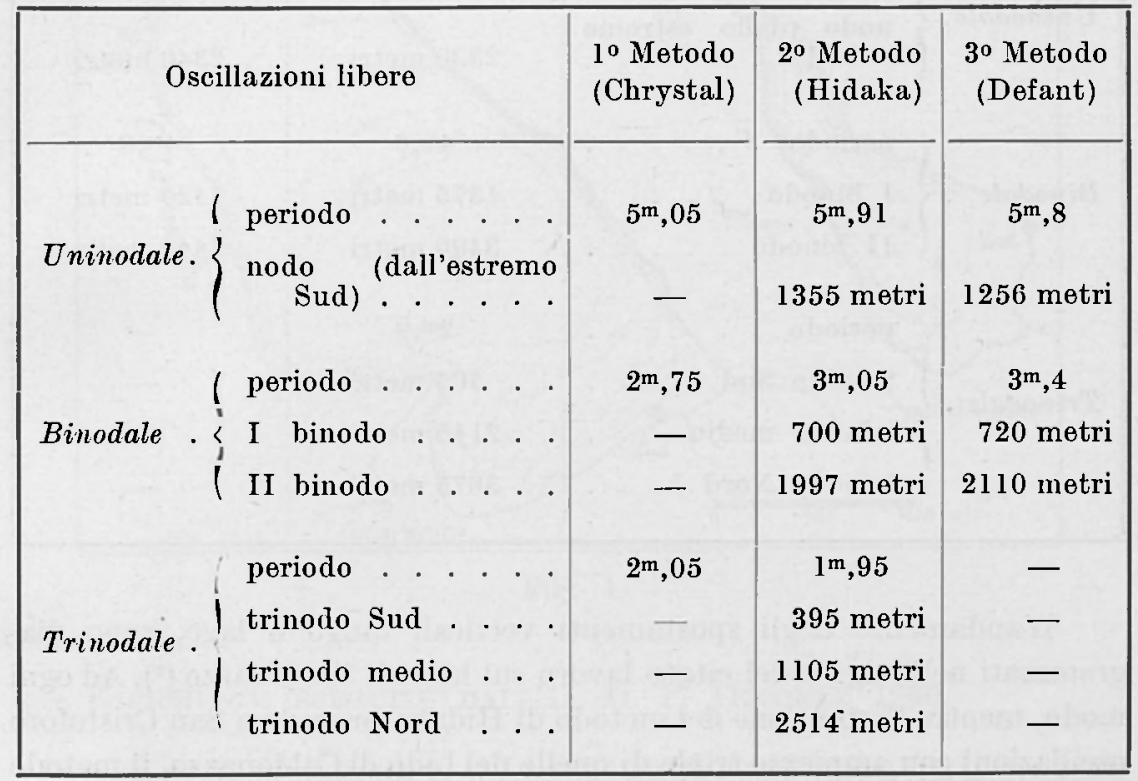

Per quanto concerne i periodi e i nodi, i risultati ottenuti per il Lago di Levico sono riassunti nella tabella $\mathrm{I}$.

$\left(^{*}\right)$ Questo lavoro è stato condotto con contributi del Consiglio Nazionale delle Ricerche. 
L'andamento delle ampiezze lungo tutto il lago di Levico, relative alle oscillazioni uninodali, binodali e trinodali è riportato in grafico, nella fig. 5 del citato lavoro. Limitatamente agli estremi - che qui solo interessano -, il metodo di Defant prevede che all'estremo Nord gli spostamenti verticali associati nell'uninodale risultano 3,8 volte quelli che si hanno all'estremo Sud, mentre l'estensione del metodo di Hidaka porta alla conclusione che le uninodali hanno sulle sponde settentrionali un'ampiezza tripla di quella che muove l'acqua a Sud.

Con riferimento al lago di Caldonazzo, la teoria porta ai risultati riassunti nella tabella II, per ciò che si riferisce ai periodi e ai nodi.

Tabella II

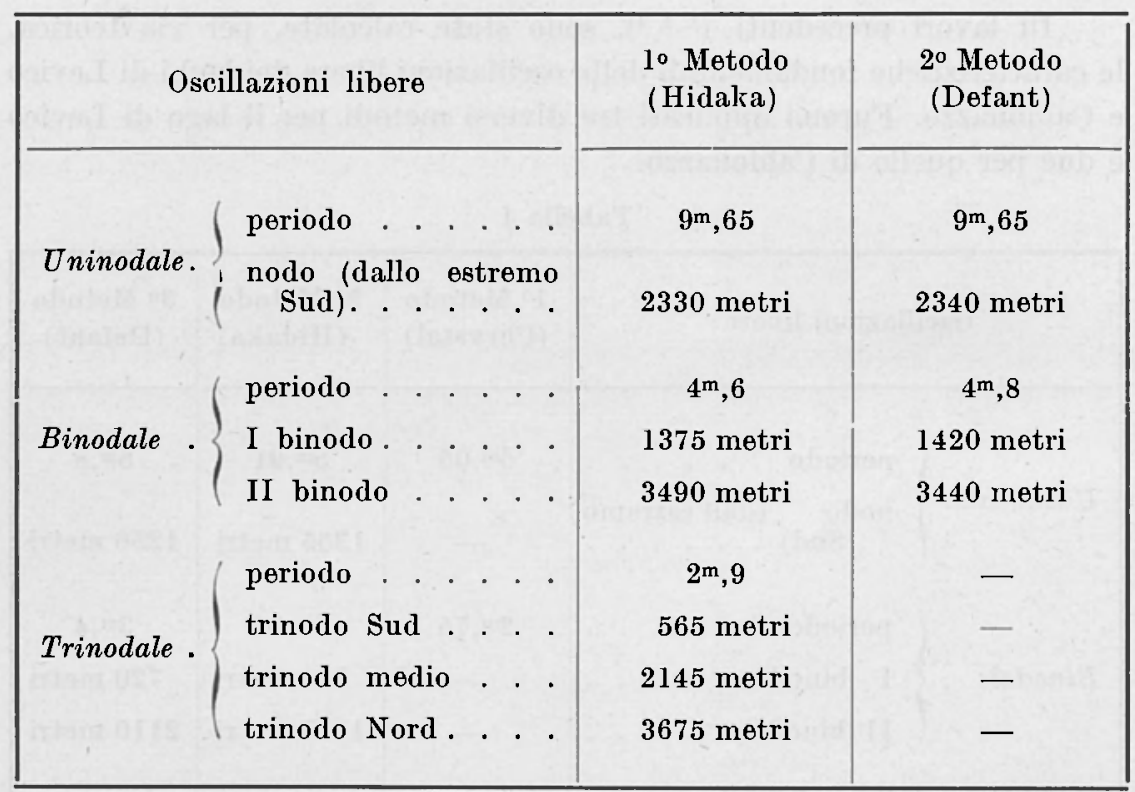

L'andamento degli spostamenti verticali lungo il lago, sono diagrammati nella fig. 4 del citato lavoro sul lago di Caldonazzo $\left({ }^{2}\right)$. Ad ogni modo, mentre l'estensione del metodo di Hidaka prevede a San Cristoforo oscillazioni con ampiezze triple di quelle del Lido di Caldonazzo, il metodo di Defant porta a ritenere le ampiezze delle uninodali nella parte Nord del lago (San Cristoforo) 2,6 volte quelle della parte sud (Lido di Caldonazzo). Nei lavori citati sono pure calcolati gli spostamenti orizzontali relativi delle acque in corrispondenza dei nodi; il controllo di questi ultimi è però rimandato ad ulteriori esperienze. 
Nelle campagne, sui risultati delle quali si riferisce, è stato fatto uso di una coppia di limnografi a galleggiante, riproducenti i movimenti verticali in vera grandezza ed opportunamente filtrati. I relativi registratori avevano - per l'occasione - uno scorrimento di $120 \mathrm{~mm} / \mathrm{h}$, particolarmente adatto per la registrazione delle oscillazioni libere dei due laghi, permettendo una sufficiente risoluzione anche per le sesse dovute allo zoccolo ("shelf-seiches »).

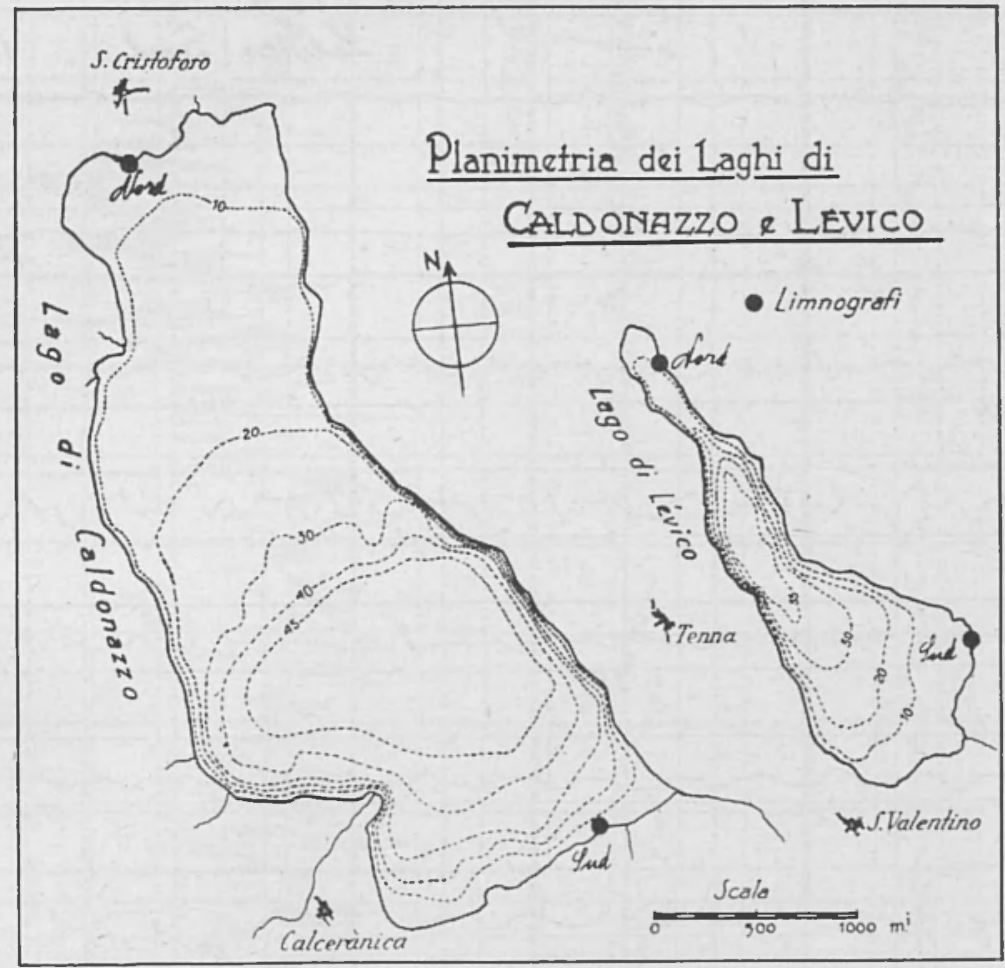

Fig. 1

\section{I. - Esperienze ESEguite DALL'11 AL 17 OtToBRe 1960.}

La settimana durante la quale fu eseguita una prima serie di osservazioni, rientrava in un lungo periodo di piogge continue: $\mathrm{i}$ laghi di Levico e Caldonazzo erano sovrabbondanti d'acque.

Il primo superava il livello normale di almeno $40 \mathrm{~cm}$, ancora più allarmante era la situazione del secondo: le sue acque infatti invadevano le spiagge oltre il bacino abituale, sommergendo le banchine e le attrez- 
zature degli stabilimenti bagni sia al Lido di Caldonazzo che a quello di San Cristoforo.

a) Osservazioni sul lago di Levico.

Fu posto un limnografo in funzione presso l'Albergo al Lago (" La Taverna "), in località sulle rive meridionali del lago (vedi fig. 1). Il

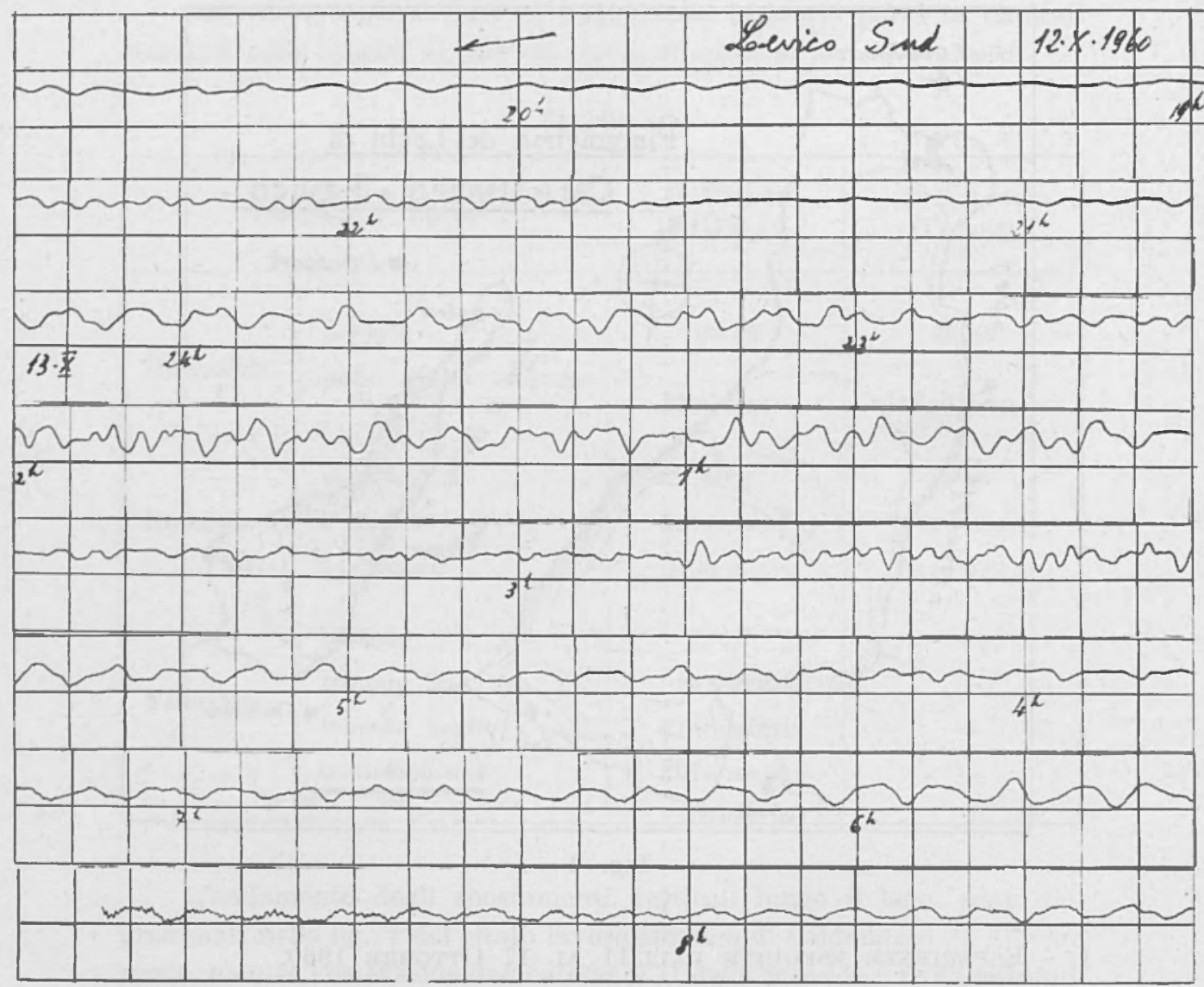

Fig. 3 - Trinodali, binodali ed uninodali sul lago di Levico (riduzione 6/10).

limnografo era filtrato, in modo da evitare la registrazione delle onde di superficie (onde progressive), provocate dal vento.

Non ostante l'avversità del tempo e alcuni inconvenienti nel sistema d'orologeria del registratore, le registrazioni ottenute il giorno 12 Ot- 




Figr. 2 - Effetti del transito sul lago di Levico di un temporale: ampia oscillazione forzata, con sovrapposte evirlenti sesse di zoccolo. Seguono, verso le $16^{\mathrm{h}} 15^{\mathrm{m}}$, aleune uninodali (ridotto a $9 / 10$ dell oririnale). 
tobre risultarono interessanti per diversi motivi: per la varietà delle oscillazioni (uninodali e multinodali) e delle cause.

Innanzitutto va notata un'ecezionale registrazione di onde forzate, determinate dal transito di un temporale. Verso le $14^{\mathrm{n}} 0 \mathrm{o}^{\mathrm{s}}$ il vento prese a soffiare sul lago provocando la registrazione di "shelf-seiches" oscillazioni libere dello strato d'acqua, che ha per fondo lo zoccolo solido, fin dove questo confina con la sca pata sommersa, che conduce alla parte più profonda del lago -. Alle $14^{\mathrm{h}} 30^{\mathrm{m}}$, proveniente da Ovest, il centro depressionario del temporale transita sul lago: immediatamente il livello di questo subisce un balzo positivo, che lo fa innalzare di circa due centimetri e mezzo in circa $5^{\mathrm{m}}$ (fig. 2). E come se il lago fosse risucchiato dal nucleo di depressione; fenomeno questo frequente, in condizioni idonee, anche su altri laghi $\left({ }^{6,7}\right)$. Il livello del lago è quindi costretto in un'onda forzata, avente uno pseudo-periodo di tre quarti d'ora circa, dopo di che il livello torna gradatamente al suo valore normale. Tale onda forzata è senz'altro da attribuire ad un'oscillazione portante della pressione atmosferica, associata al temporale, avente un analogo pseudoperiodo. Sovrapposta a tale oscillazione fondamentale è tutta una lunga serie di oscillazioni minori, tra le quali prevalgono quelle di $1^{\mathrm{m}}$ di periodo, particolarmente ampie, e di $30^{\mathrm{s}}$ : sesse dello zoccolo o sesse forzate? Probabilmente le une e le altre; oscillazioni forzate di $1^{\mathrm{m}}$ circa e ${ }^{\text {shelf- }}$ seiches " di un periodo di circa $30^{\text {s. }}$.

Passato il temporale e caduto il vento, il lago ha cominciato una serie di oscillazioni libere uninodali, alterate da residue perturbazioni dello zoccolo: il periodo predominante si aggira sui $6^{\mathrm{m}}$.

Verso le $18^{\mathrm{n}} 10^{\mathrm{m}}$ si sovrappongono alle deboli oscillazioni uninodali, altrettanto deboli oscillazioni binodali.

Dopo poco più di un'ora di relativa calma, il lago ha ripreso i suoi lievi movimenti; alle $21^{1} 32^{\mathrm{m}}$ inizia una serie di onde trinodali $\left(T \cong 2^{\mathrm{m}}\right)$, sovrapposte a binodali $\left(2^{\mathrm{n}} 8^{\mathrm{m}}-3^{\mathrm{m}}\right)$, finchè verso le $23^{\mathrm{n}}$ insorgono pure gruppi di uninodali, rendendo la registrazione alquanto irregolare. Intorno alle $24^{\mathrm{h}}$, le uninodali prevalgono chiaramente con periodi di $6^{\mathrm{m}}$ netti. Quindi, nuova sovrapposizione di binodali $\left(2^{\mathrm{m}} \mathrm{s}\right)$, che prevalgono a loro volta verso i tre quarti del 13 Ottobre, dopo di che la registrazione torna a complicarsi per la sovrapposizione di oscillazioni libere uninodali, binodali e trinodali $\left(1^{\mathrm{m} 9 \mathrm{~s}}-2^{\mathrm{m}}\right)$, queste ultime particolarmente chiare dalle $2^{\mathrm{n}} 05^{\mathrm{m}}$ alle $2^{\mathrm{h}} 50^{\mathrm{m}}$ ca.

Verso le $4^{\mathrm{h}}$ inizia una lunga serie di uninodali $\left(6^{\mathrm{m}}\right)$, qua e là disturbate da binodali, che dura ininterrotta fino alle $9^{\mathrm{n}} 45^{\mathrm{m}}$ circa del mattino (fig. 3). 
Oltre alla registrazione di chiari esempi di uninodali, il cui periodo è in perfetto accordo con quello previsto dalla teoria, questa prima serie di osservazioni ha consentito la sorpresa della registrazione di onde binodali e trinodali, pure previste dalla teoria ma il cui naturale insorgere ritenevo comunque ben più difficile.

Restava ancora da controllare un altro importante aspetto delle teorie idrodinamiche, quello generalmente trascurato del rapporto delle ampiezze registrate ai due estremi del lago. La teoria prevedeva, per il lago di Levico, ampiezze di oscillazione nettamente superiori per le uninodali nel lato Nord del lago. Tale controllo richideva la contemporanea registrazione effettuata ai due estremi del lago. L'accesso alla estremità settentrionale del lago, reso poco agevole da litoranei disagiati sentieri o da una erta, scoscesa stradicciola staccantesi dalla sovrastante strada nazionale Trento-Bassano, risultava pressoché proibitivo a cagione del tempo pessimo.

Il controllo delle ampiezze fu per tanto rimandato ad una successiva campagna di misure.

\section{b) Osservazioni sul lago di Caldonazzo.}

Già a mezzogiorno del 13 Ottobre un limnografo era in funzione presso il lago di Caldonazzo, sulle sponde meridionali (vedi fig. 1). Nella notte ebbe a registrare una lunga serie di oscillazioni uninodali, di piccola ampiezza, ma molto regolari. Fino alle $9^{\mathrm{h}}$ del 14 , lo scorrimento del registratore fu di soli $4 \mathrm{~cm} / \mathrm{h}$.

Messo lo scorrimento a $12 \mathrm{~cm} / \mathrm{h}$, verso le $11^{\mathrm{h}}$ del 14 Ottobre si ha la ripresa della registrazione di piccole oscillazioni uninodali, aventi un periodo di $10^{\mathrm{m}}$ esatti (fig. 4). Verso le $14^{\mathrm{h}}$ un vento leggero muove la superficie del lago; intorno alle $16^{\mathrm{h}}$ e alle $17^{\text {h }}$ si ha la registrazione di chiare oscillazioni trinodali (periodo $3^{\mathrm{m}}$ circa).

Nella tarda sera la pioggia ripiglia a cadere, provocando un'ulteriore ascesa del livello del lago, che continua ininterrotta durante tutta la notte. Dopo lievi oscillazioni uninodali, registrate dalle $20^{\mathrm{h}}$ alle $22^{\mathrm{h}}$ - periodo $10^{\mathrm{m}}$ esatti - verso le $22^{\mathrm{h}} 30^{\mathrm{m}}$ insorge un piccolo gruppo di oscillazioni binodali (periodi fra $4^{\mathrm{m}}, 6$ e $4^{\mathrm{m}}, 8$ ) (fig. 5).

Quel gruppetto di binodali è l'inizio di un lungo seguito di oscillazioni che - sotto certi aspetti - costituisce la parte più interessante delle registrazioni ottenute in quell'occasione, sul lago di Caldonazzo. È infatti un alternarsi, e un sovrapporsi di onde binodali e trinodali: le prime raggiungono massimi nettissimi fra le $2^{\text {h }}$ e le $3^{\text {h }}$, con periodi medi di $4^{\mathrm{m}} 6^{\mathrm{s}}$; le seconde si rendono particolarmente evidenti fra le $3^{\mathrm{h}}$ e le $4^{\mathrm{h}}$. 


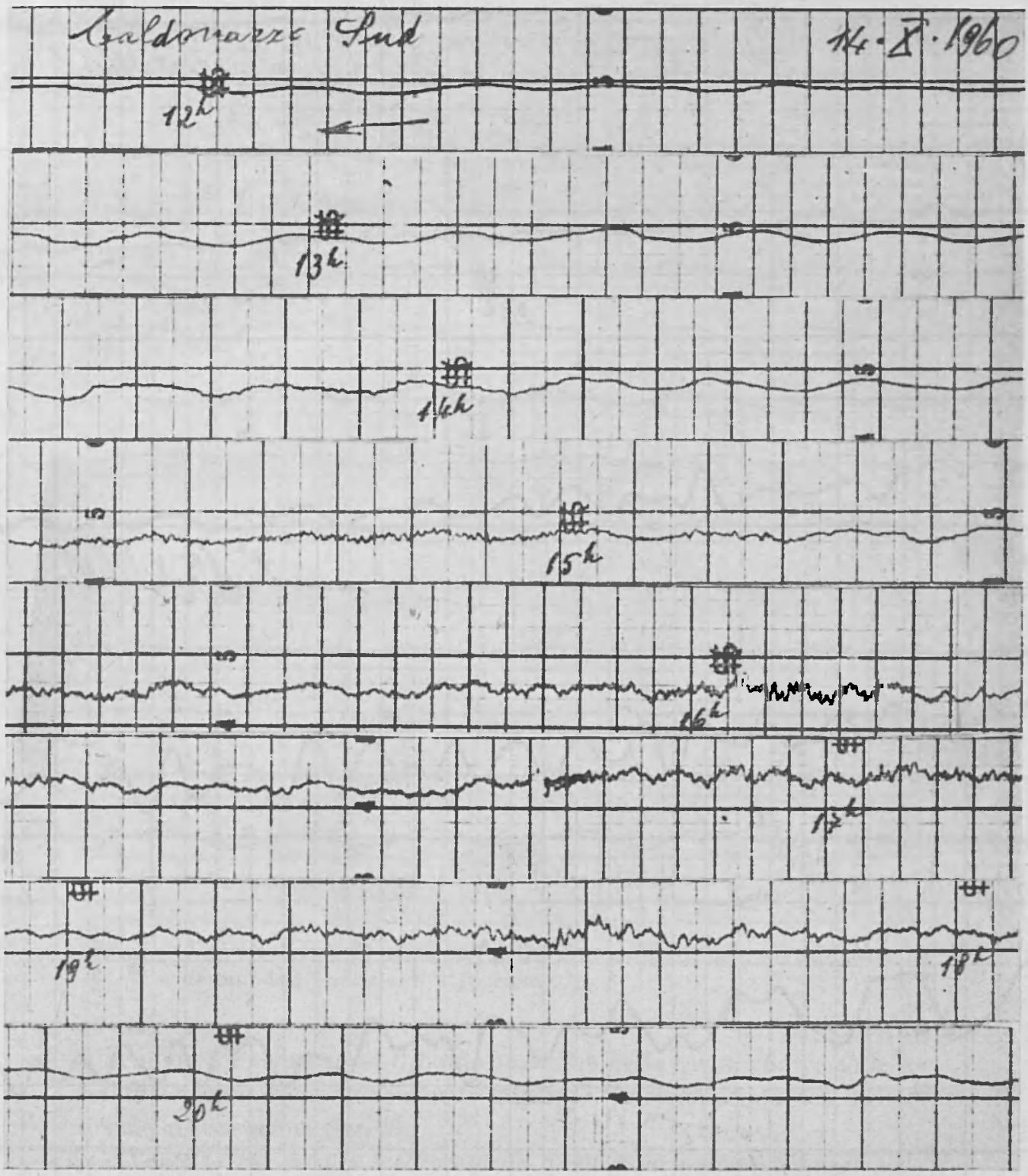

Fig. 4 - Lieve attività del lago di Caldonazzo (riva meridionale); perturbazioni per vento dalle $14^{\mathrm{h}}$ alle $19 \mathrm{~h}$, sovrapposte ad uninodali di piccola ampiezza. 


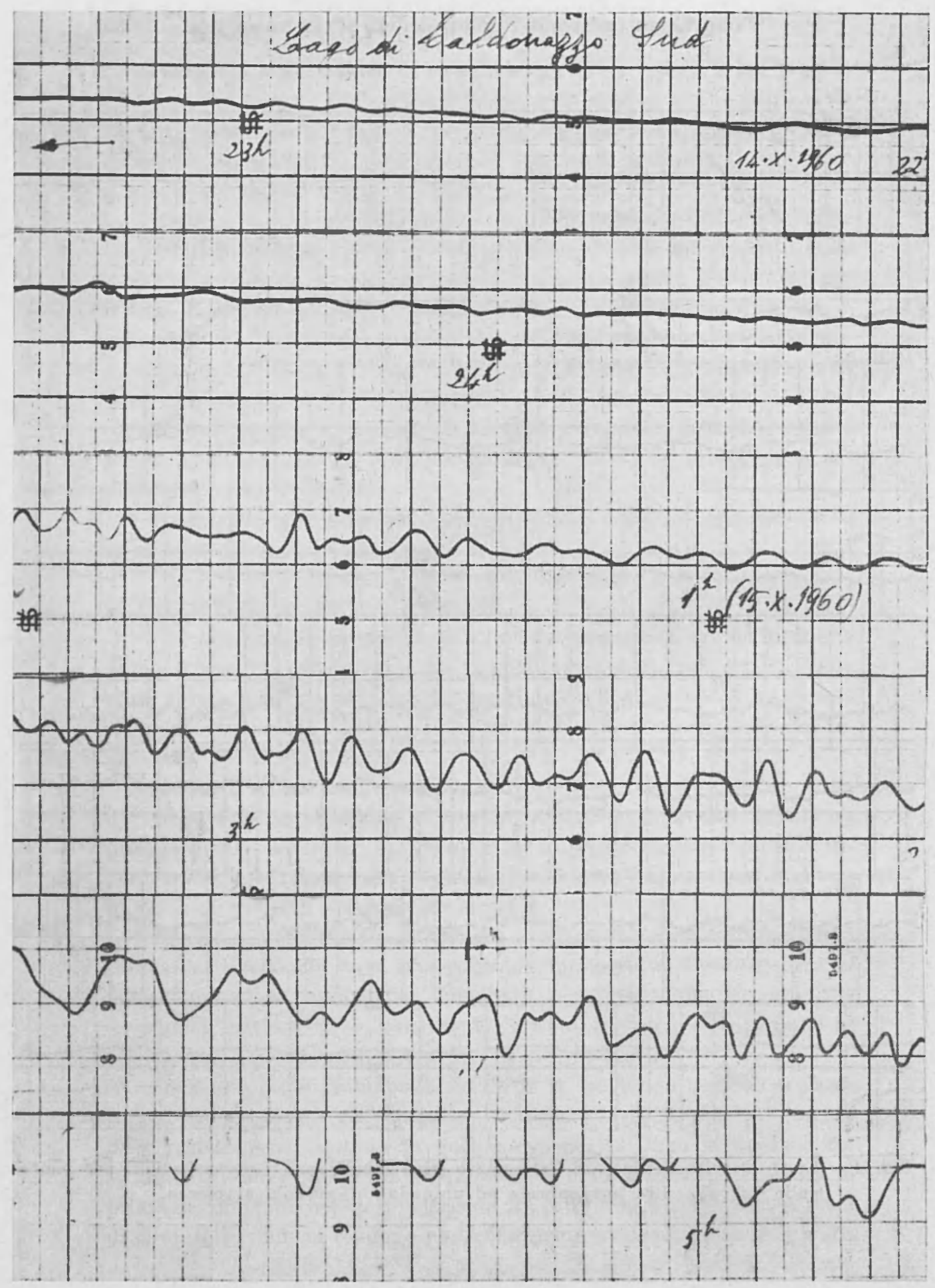

Fig. 5 - Eccezionale registrazione a Caldonazzo Sud, con atmosfera calma: binodali e trinodali precedono l'insorgere di evidenti uninodali. 
A completare ed a complicare il ritmo del lago, verso le $3^{\mathrm{n}} 40^{\mathrm{m}}$ cominciano ad apparire le uninodali che raggiungono subito sensibili ampiezze, e tendono a prevalere nella gara, sebbene fortemente contrastate dal perdurare delle binodali (dicrote) e dalle trinodali. Nel frattempo, il livello del lago era andato via via salendo $\left(7 \mathrm{~cm}\right.$ in $\left.10^{\mathrm{h}}\right)$, costringendo



Fig. 6 - Lieve attività di uninodali a Caldonazzo Sud, con sovrapposizione di binodali (riduzione $8 / 10$ cirea).

la pennina scrivente verso il bordo superiore della carta, che verso le $6^{\text {h }}$ del 15 Ottobre viene decisamente superato, interrompendo così l'eccezionale registrazione (fig. 5).

Verso le $14^{\mathrm{h}}$ il lago è sorpreso da oscillazioni uninodali quasi pure $\left(10^{\mathrm{m}}\right)$, che rapidamente si attenuano. Dalle $19^{\mathrm{h}} 50^{\mathrm{m}}$ alle $20^{\mathrm{h}} 15^{\mathrm{m}}$ si ha la registrazione di un gruppo di binodali $\left(4^{\mathrm{m}} 7^{\mathrm{s}}\right)$, cui segue, alle $20^{\mathrm{n}} 25^{\mathrm{m}}$ un gruppetto di trinodali $\left(3^{\mathrm{m}}\right)$. Intorno alle $22^{\mathrm{n}}$ riprendono le uninodali, di piccola ampiezza, che durano, pressochè ininterrottamente, fino al- 
le $7^{\mathrm{h}}$ del 16 Ottobre. Il livello del lago, nel frattempo, è andato aumentando di altri $4 \mathrm{~cm}$.

Fino alla tarda sera del 16, l'attività ritmica del lago fu molto limitata: solo qualche lieve gruppo di uninodali dalle $16^{\mathrm{h}}$ alle $20^{\mathrm{h}}$. Prece-

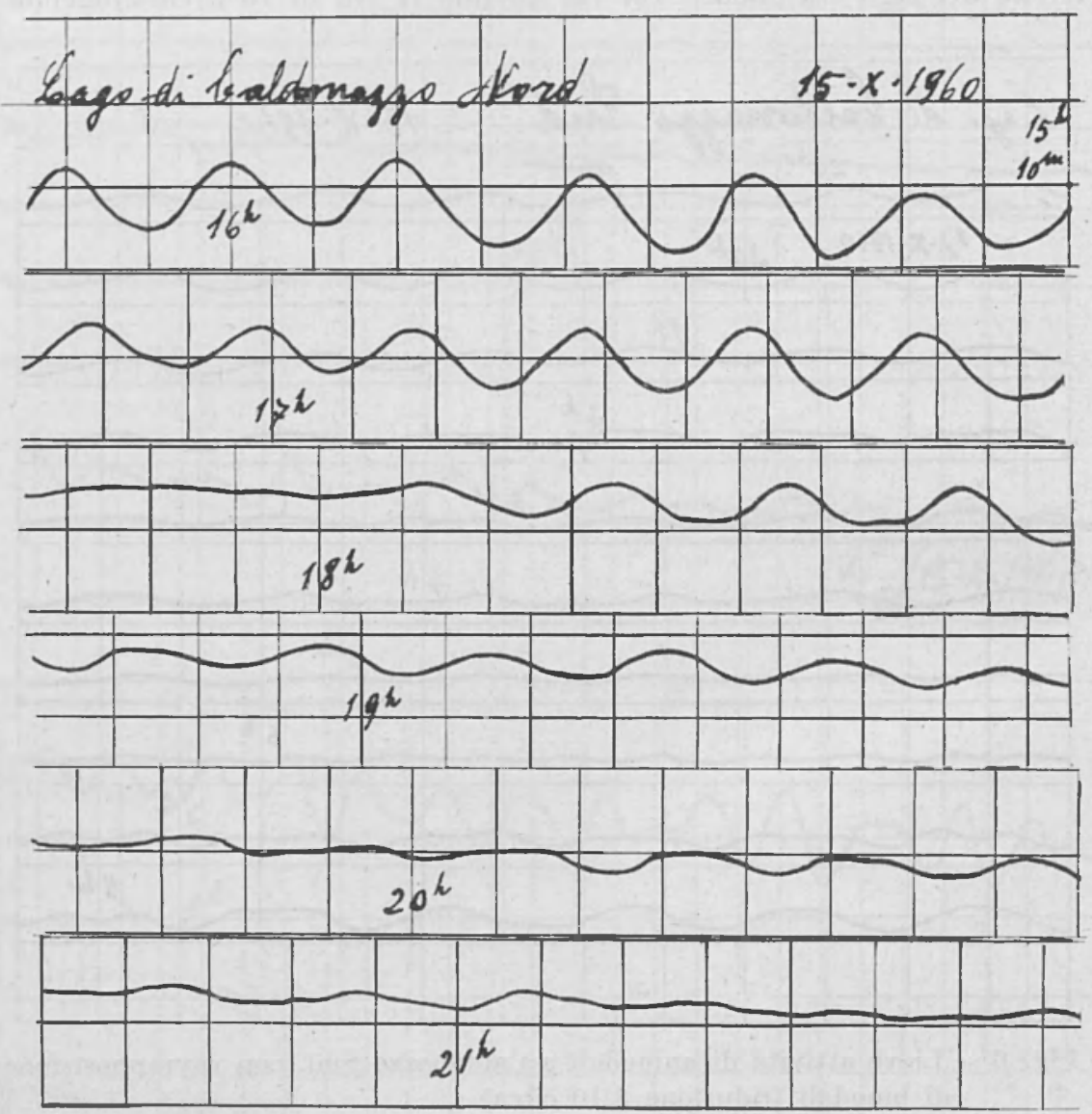

Fig. 7 - Chiari esempi di uninodali sul lato Nord del lago di Caldonazzo (riduzione $9 / 10$ ).

duta - come sovente si verifica - da debolissime binodali, verso le $22^{\mathrm{h}} 30^{\mathrm{m}}$ inizia una serie di uninodali, che continua ininterrotta (sia pure con piccola ampiezza) fino alle $6^{\text {h }}$ circa del 17 Ottobre (fig. 6). Il periodo è sempre di $10^{\mathrm{m}}$ circa. Dalle $3^{\mathrm{h}}$ alle $5^{\mathrm{h}}$ si sovrappongono alle uninodali alcune binodali (dicrote). Una nuova serie di uninodali, anche questa volta preceduta da un persistente gruppo di binodali $\left(4^{\mathrm{m}}, 8-5^{\mathrm{m}}\right)$ inizia 
verso le $7^{\mathrm{h}} 30^{\mathrm{m}}$ del 17 , e raggiunge subito ampiezze di qualche millimetro. La registrazione fu dovuta interrompere, essendo venuta l'ora della partenza (fig. 6).

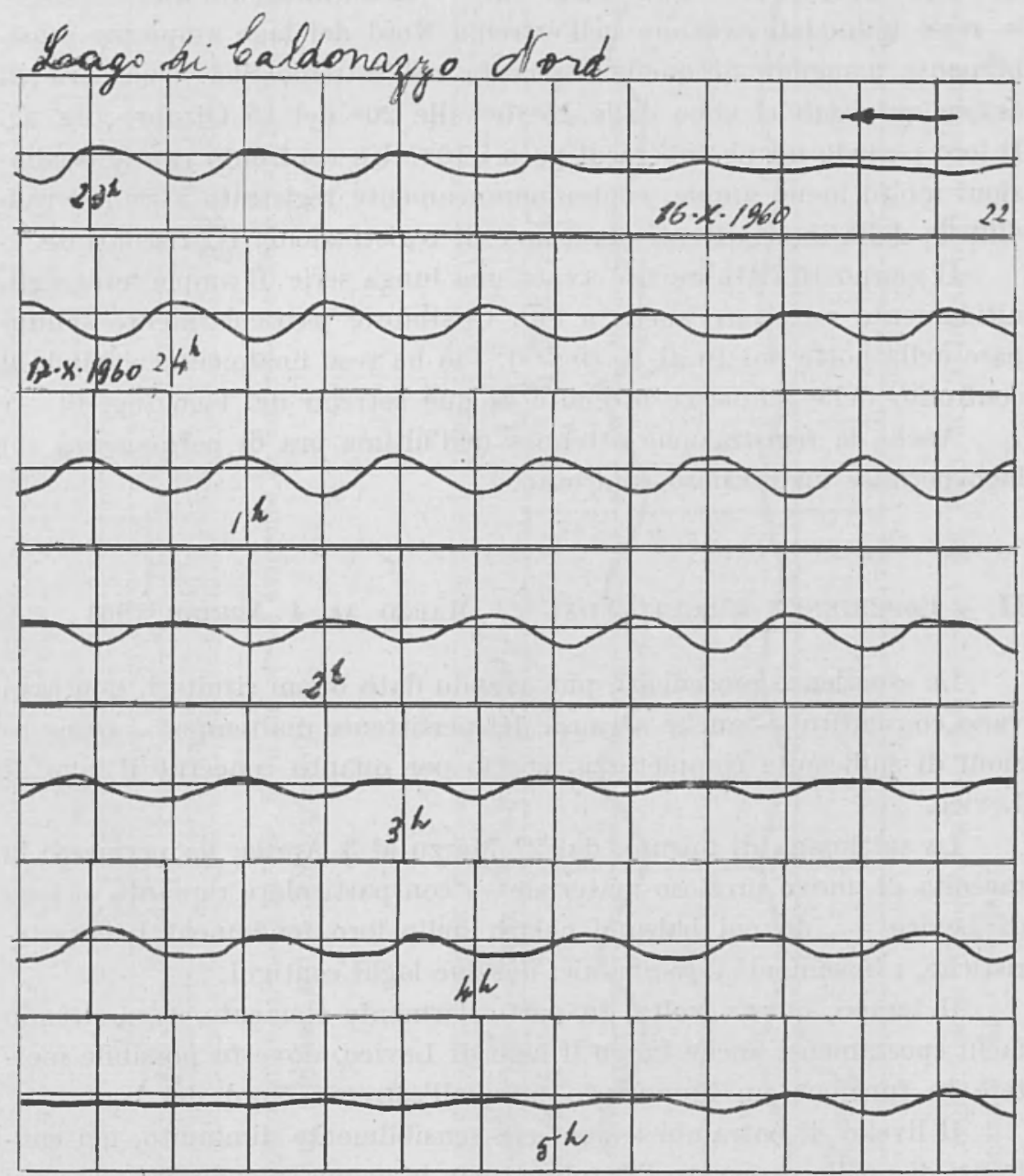

Fig. 8 - Uninodali di piccola ampiezza a Caldonazzo Nord (ridotta a 8/10 circa).

Sulle sponde settentrionali del lago, a San Cristoforo, presso l'elegante stabilimento bagni ivi esistente (fig. 1), il limnografo che, in quei giorni, si era cercato di mettere in contemporanea funzione, rifiuto caparbiamente di registrare: l'orologeria del registratore, infatti, accusò 
frequentissimi arresti e a ben poco valse l'abilità del tecnico per tentare di ottenere registrazioni di una certa continuità.

Ad ogni modo, si è avuta ugualmente qualche bella registrazione, dalla quale apparve subito chiaro che - conformemente alla teoria le sesse uninodali avevano nell'estremo Nord del lago ampiezza sensibilmente maggiore di quella osservata nell'estremo Sud. Una serie di ampie uninodali si ebbe dalle $15^{\mathrm{h}} 10^{\mathrm{m}}$ alle $20^{\mathrm{h}}$ del 15 Ottobre (fig. 7 ). Il loro periodo medio supera di poco i $10^{\mathrm{m}}$. Un confronto con le oscillazioni molto meno ampie, contemporaneamente registrate a Sud, è reso difficile dalle intermittenti mancanze di registrazione, ivi riscontrate.

Il giorno 16 Ottobre si è avuta una lunga serie di ampie uninodali, nitidamente registrate anche a San Cristoforo, particolarmente sviluppate nella notte dal 16 al 17 (fig. 8). Ciò ha reso finalmente possibile il confronto delle ampiezze ottenute ai due setremi del lago (fig. 9).

Anche la registrazione ottenuta nell'ultima ora di permanenza sul lago permise un prezioso confronto.

\section{II. - ESPERIENZe ESEgUtTe DAL 27 MARzo AL 4 APRILE 1961}

Le esperienze precedenti, pur avendo dato buoni risultati, non avevano consentito - anche a causa del persistente maltempo - osservazioni di sufficiente completezza, specie per quanto concerne il lago di Levico.

La settimana di misure, dal 27 Marzo al 3 Aprile, ha permesso la raccolta di nuovo prezioso materiale - con particolare riguardo al lago di Levico - , da cui balzano chiari, nelle loro fondamentali caratteristiche, i lineamenti idrodinamici dei due laghi contigui.

Il tempo, questa volta, fu particolarmente clemente, permettendo facili spostamenti anche lungo il lago di Levico, dove fu possibile mettere in funzione un limnografo pure all'estremo Nord del lago.

Il livello di entrambi i laghi era sensibilmente diminuito, nei confronti di quello osservato l'Ottobre 1960, le acque essendo rientrate nei limiti dell'alveo normale.

\section{a) Osservazioni sul lago di Levico.}

Un limnografo ha cominciato a funzionare presso il porticciuolo dell'albergo al Lago ("La Taverna »), la sera del 27 Marzo, alle $19^{\mathrm{h}} 30^{\mathrm{m}}$. Dopo qualche sporadico esempio di uninodale (verso le $21^{\mathrm{h}} 30^{\mathrm{m}}$ ) ed altre 


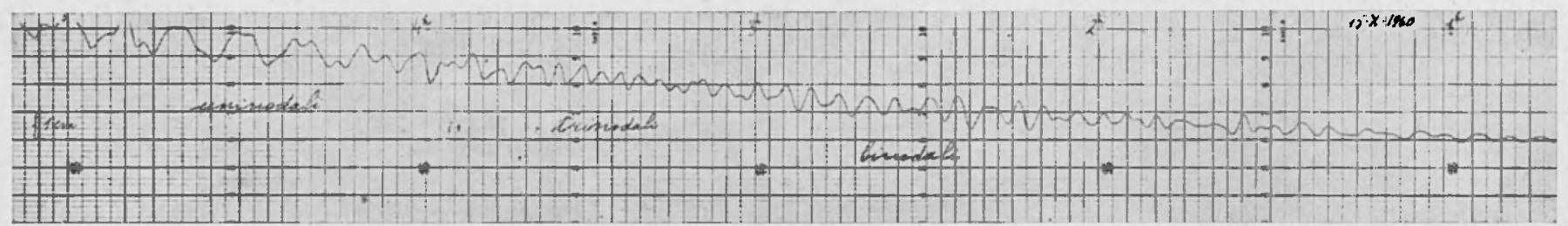

Fig. 5a - Tratto della registrazione, di eui alla Fig. 5, in cui riesce evidente la successione delle multinodali, che precedono le uninodali (ridotto a $1 / 3$ dell'originale).

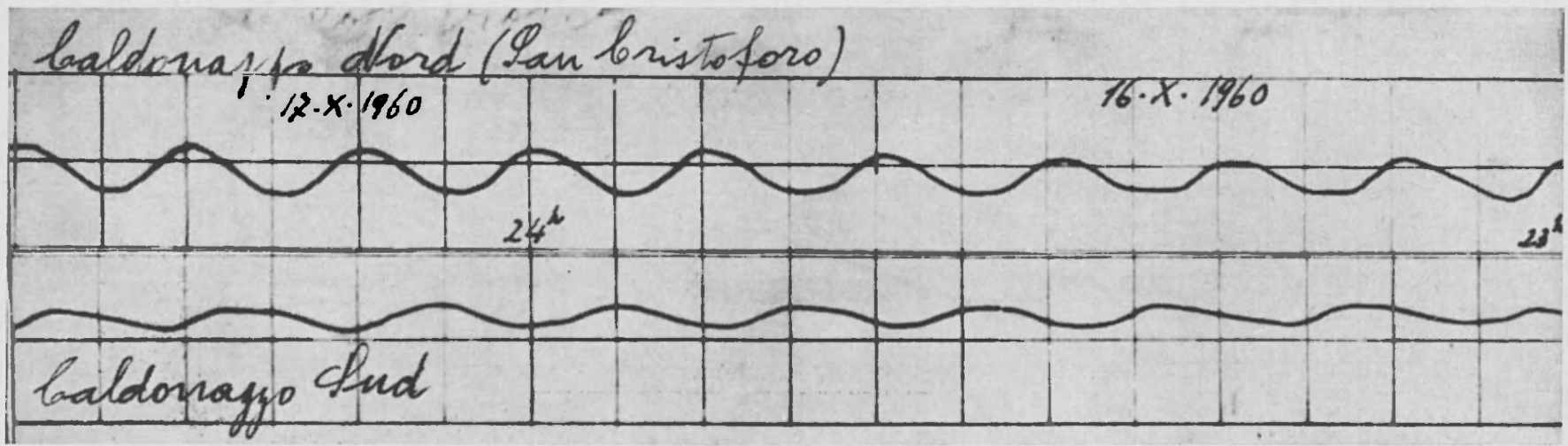

Fig. 9 - Contemporanea registrazione di uninodali sui lati estremi del lago di Caldonazzo (è manifesto il netto prevalere dell ampiezza a San Cristoforo). 


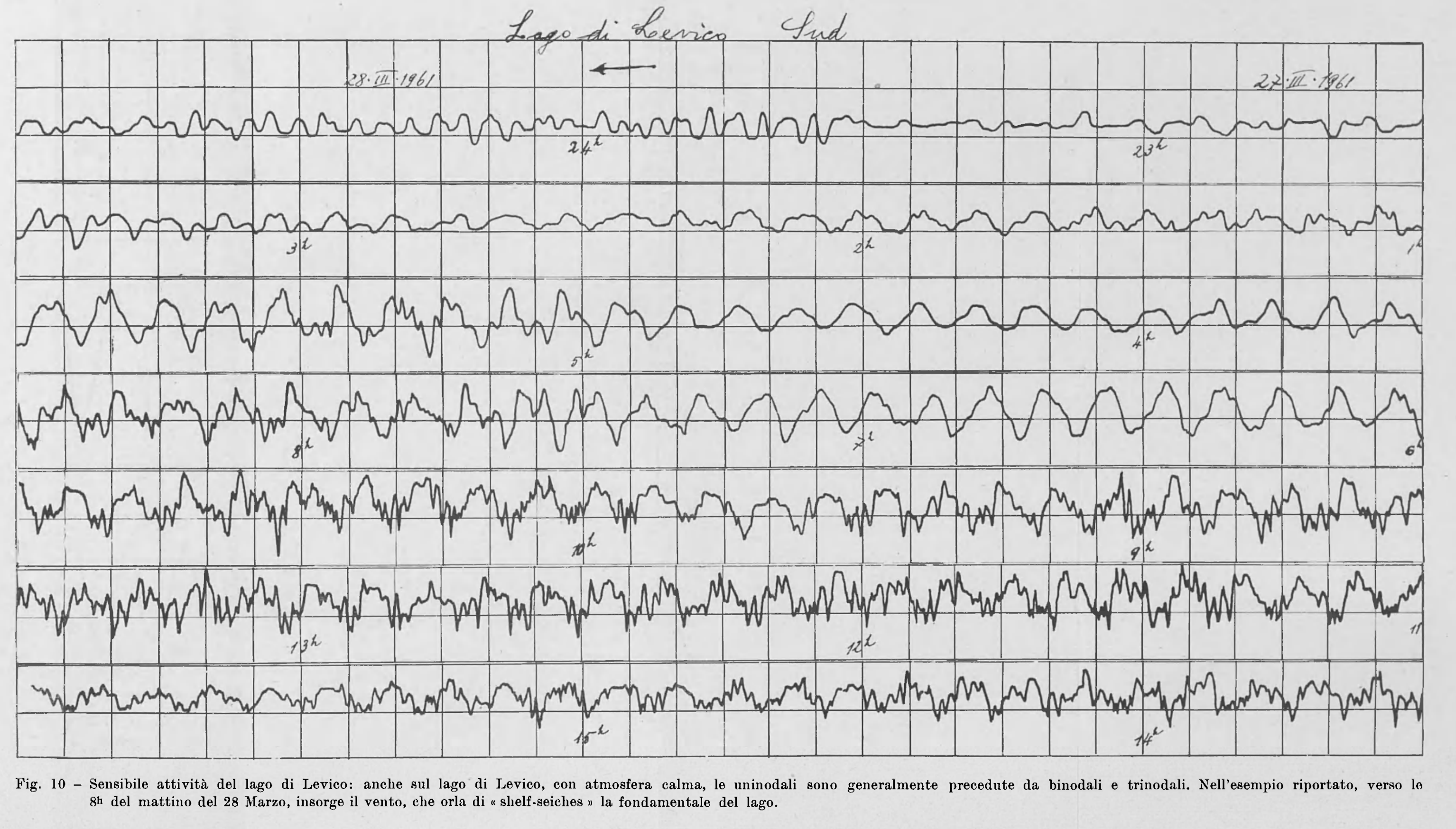




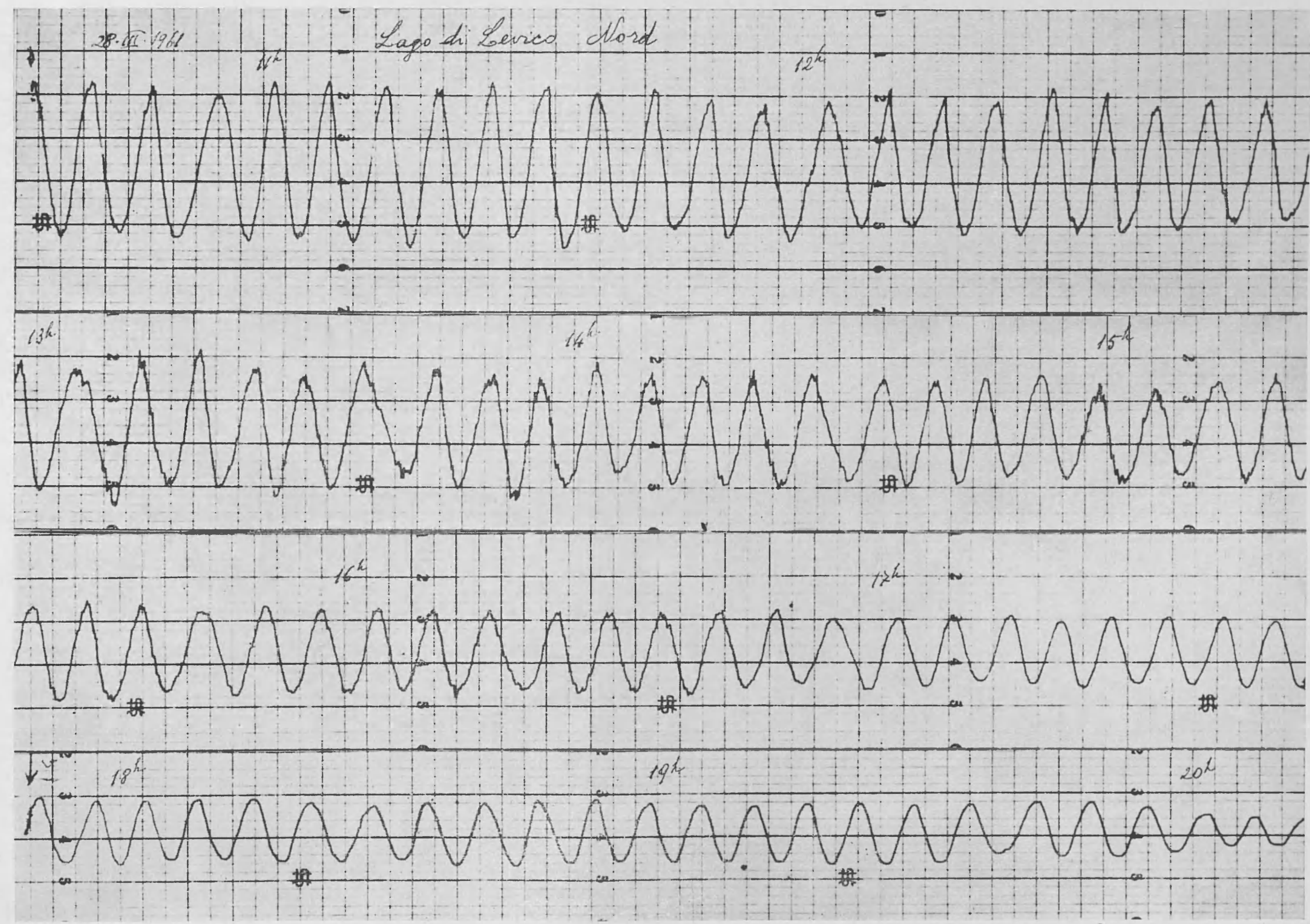

Fig. 11 - Registrazione di ampie uninodali sul lato Nord del lago di Levico. 




Fig. 12 - Attività normale di uninodali nel lato Nord del lago di Levico (ridotta a circa $9 / 10$ dell originale).

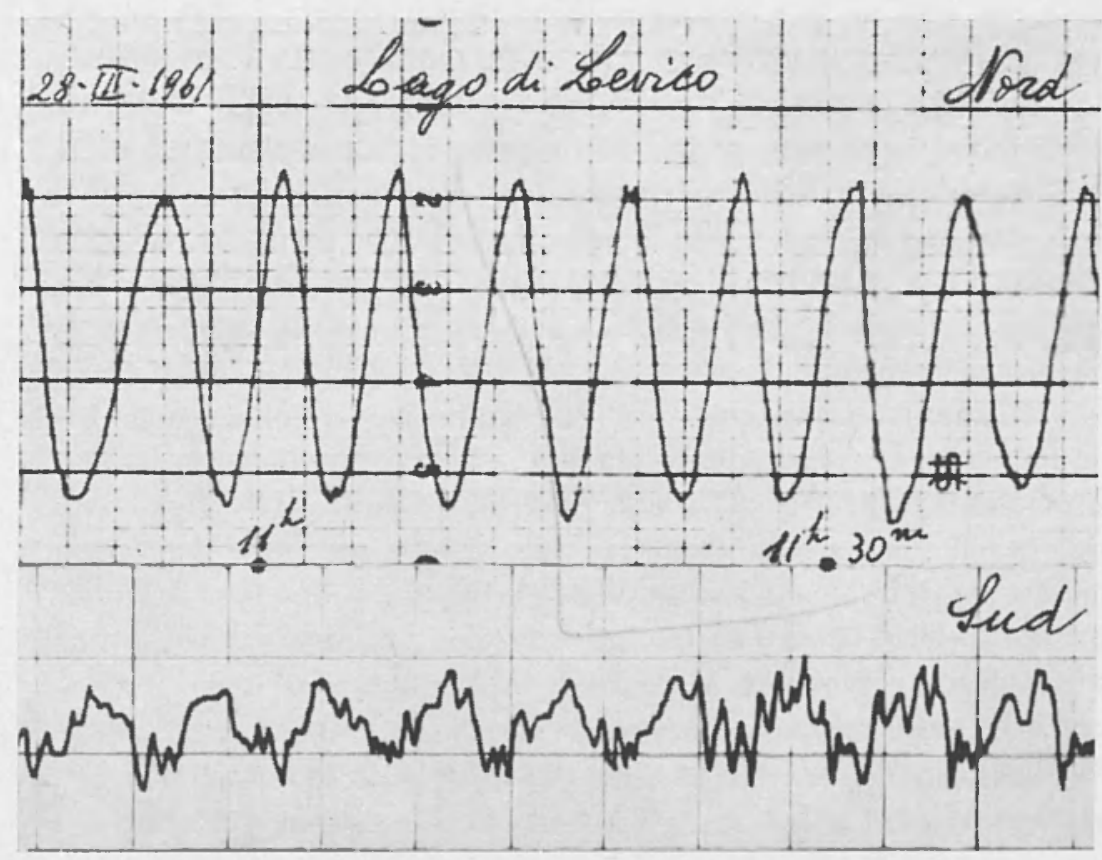

Fig. 13 - Contemporanea registrazione di uninodali ai due estremi del lago di Levico: la parte settentrionale oscilla con ampiezze pressochè quadruple di quelle che si osservano nella parte meridionale. 
multinodali fra le $22^{\mathrm{h}}$ e le $23^{\mathrm{h}}$, alle $23^{\mathrm{h}} 28^{\mathrm{m}}$, bruscamente, il lago inizia a muoversi al ritmo di binodali e trinodali, che si alternano e si sovrappongono fino alle $00^{\mathrm{h}} 40^{\mathrm{m}}$ ca. del $28 \mathrm{Marzo}$, quando cominciano a insorgere le uninodali, aventi un periodo di $6^{\mathrm{m}}$. Le binodali tornano a prevalere verso le $3^{\text {h }}$, dando luogo a registrazione di dicrote, seguite quindi da uninodali, pressochè pure, dalle $3^{\mathrm{h}} 50^{\mathrm{m}}$ alle $4^{\mathrm{h}} 45^{\mathrm{m}}$. Esempi di ampie binodali ( $3^{\mathrm{m}}$ ca.), e quindi di dicrote, si hanno dalle $4^{\mathrm{h}} 45^{\mathrm{m}}$ alle $5^{\mathrm{h}} 30^{\mathrm{m}}$, con sovrapposizione di "shelf-seiches" (1 $\mathrm{m}$ ca. di periodo) in coincidenza al soffiar di un leggero vento, mentre dalle $5^{\mathrm{h}} 50^{\mathrm{m}}$ le uninodali tornano a prevalere ampie, conservando un periodo di $6^{\mathrm{m}}$ esatti. Alle $6^{\mathrm{h}} 30^{\mathrm{m}}$ il vento si va facendo piuttosto teso e soffia sulla parte meridionale del lago fino al tardo pomeriggio, con la conseguenza di orlare con ampie " shelf-seiches " le persistenti uninodali (fig. 10).

Il 29, nella parte meridionale del lago, i moti stazionari sono di piccola entità. Solo verso le $16^{\mathrm{h}} 15^{\mathrm{m}}$, il vento, riprendendo a soffiare leggero, provoca la registrazione di una lunga serie di "shelf-seiches", in cui prevalgono $i$ periodi di circa $1^{m}$; seguono debolissime uninodali.

Il limnografo che, nel frattempo, era stato sistemato nella parte Nord del lago (fig. 1), per un piccolo incidente al registratore durante la notte dal 27 al 28 non aveva funzionato. Quando finalmente, alle $10^{\mathrm{h}} 35^{\mathrm{m}}$ del 28 riprese a funzionare regolarmente, sorprese la parte settentrionale del lago animata da vistose oscillazioni uninodali, ben più ampie di quelle che contemporaneamente venivano registrate a sud. Il loro periodo medio è sempre $6^{\mathrm{m}}$, ma si registrano sovente periodi dell'ordine di $5^{\mathrm{m}}, 8-5^{\mathrm{m}}, 9$. Con l'ampiezza registrata all'inizio del funzionamento del limnografo, le uninodali dovevano alternarsi da parecchie ore, come è provato dall'ampiezza proporzionalmente ridotta ma sostenuta dalle oscillazioni registrate a Sud e dalle oscillazioni registrate a Nord dalle $7 \mathrm{~h} 10^{\mathrm{m}}$ alle $7 \mathrm{~h} 30^{\mathrm{m}}$, in un tratto di funzionamento regolare. Ad ogni modo, la loro ampiezza va smorzandosi lentamente e sono ancora sensibili alle $21^{\mathrm{h}} 40^{\mathrm{m}}$, quando il limnografo subisce un nuovo arresto (fig. 11). Rimesso in moto alle $8^{\mathrm{n}} 18^{\mathrm{m}}$ del 29 , il limnografo settentrionale riprende a registrare uninodali, con periodi lievemente inferiore ai $6^{\mathrm{m}}\left(5^{\mathrm{m}}, 8\right)$; e la registrazione continua fino alle $23^{\mathrm{h}}$, per riprendere, con piccola ampiezza, verso l' $^{1 / 1}$ del 30 Marzo. Il periodo prevalente è $5^{\mathrm{m}}, 9-6^{\mathrm{m}}$. Nella zona Nord del lago le uninodali vengono registrate pressochè in continuità, l'attività normale essendo come da fig. 12 .

Dal confronto delle uninodali registrate contemporaneamente a Nord e a Sud del lago (figg. 13, 14) risulta che, in media, le ampiezze di quelle 
della parte settentrionale del lago sono per lo meno 3,5 volte quelle osservate nella parte meridionale, più larga.

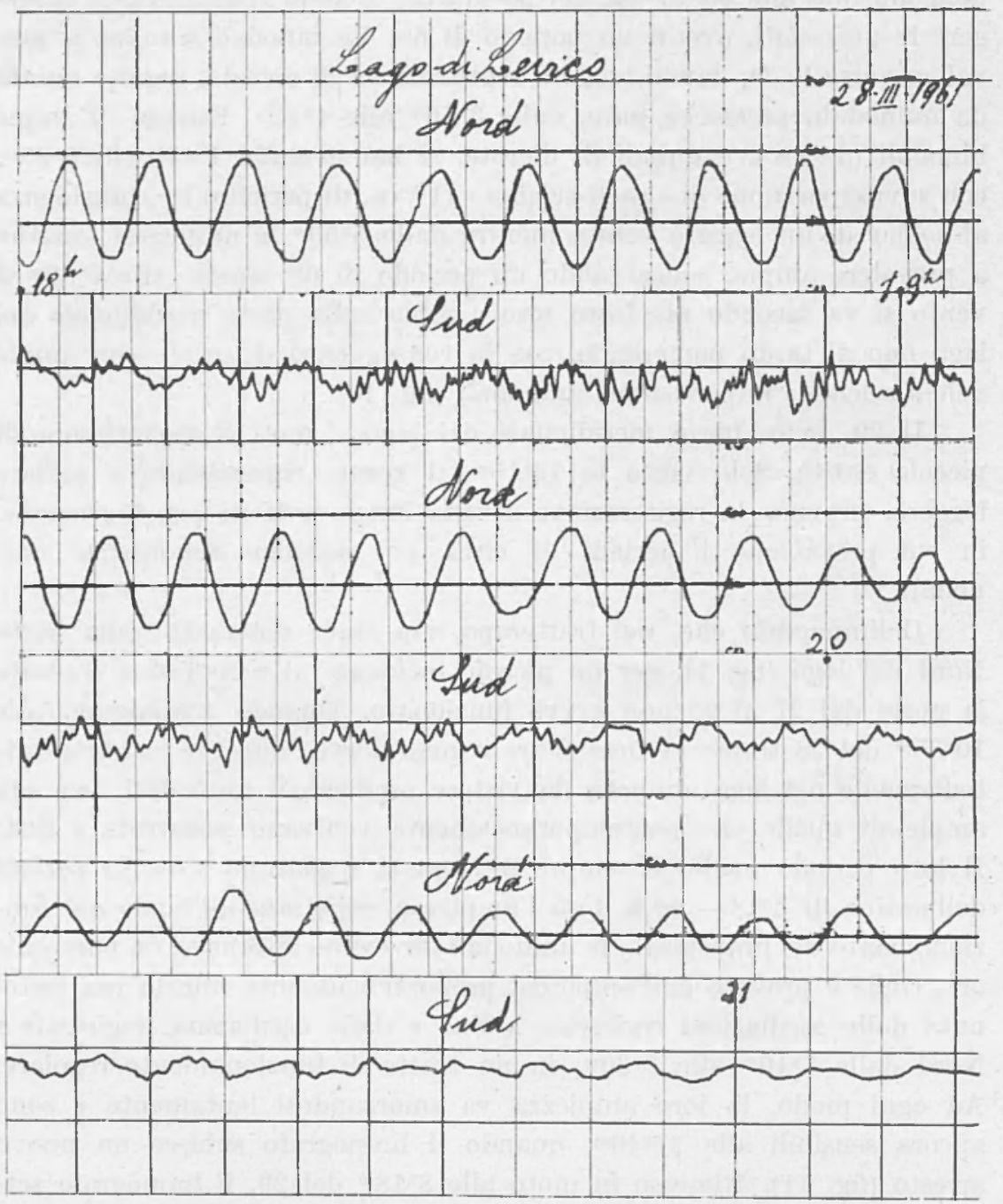

Fig. 14 - Contemporaneo alternarsi di uninodali a Nord e a Sud del lago di Levico; con manifesta differenza di ampiezze, conformemente alla teoria (riduzione a $8 / 10$ ).

Circa le oscillazioni libere del lago di Levico, si riteneva ( $\left.{ }^{4}\right)$ che esse fossero di piccola ampiezza, poco frequenti e poco persistenti. Abbiamo visto che ciò non corrisponde a verità. Questa erronea conclusione è 
forse da attribuirsi all'aver limitato le osservazioni alla parte sud del lago, ignorando che - conformemente alle indicazioni della teoria $\left(^{1}\right)$ 一, nella parte settentrionale del lago l'ampiezza delle uninodali è circa quattro volte quella osservata sulla riva meridionale; inoltre, tale oscillazione persiste a Nord pressochè ininterrottamente, passando pressochè inosservata a Sud, in caso di piccole ampiezze.

\section{b) Osservazioni sul lago di Caldonazzo.}

Il 30 Marzo, alle $10^{\mathrm{h}} 30^{\mathrm{m}}$ uno dei limnografi, tolto dalle sponde meridionali del lago di Levico, già funzionava sul lago di Caldonazzo, nella parte Sud, presso il Lido (v. fig. 1).



Fig. 15 - Media attività di uninodali a Caldonazzo Sud, con sovrapposizione di "shelf-seiches" (riduzione a 9/10).

Si hanno in prevalenza oscillazioni uninodali (periodo $10^{\mathrm{m}}$ ca.), cui si sovrappongono delle binodali (dicrote), come si verifica dalle $15^{\text {h }}$ alle $19^{\text {n }}$ (fig. 15). Alternata da periodi di quiete, le uninodali vengono registrate fino al $1^{\circ}$ Aprile (fig. 16).

Alle $16^{\mathrm{h}} 20^{\mathrm{m}}$ del 31 Marzo anche l'altro limnografo funzionava sul lago di Caldonazzo, al Lido di San Cristoforo, nel lato Nord (fig. 1).

Esso trova le acque già in moto sul ritmo delle uninodali, le quali continuano, con lievi variazioni d'ampiezza, pressochè ininterrottamente 
durante i quattro giorni di registrazione, compiuti in questa località. Vedansi le lunghe serie di uninodali indisturbate, riprodotte nelle figure 17 e 19 .



Fig. 16 - Lieve attività a Caldonazzo Sud (8/10 dell'originale).

Dalle $17^{\mathrm{h}}$ ca. alle $19^{\mathrm{h}} \mathrm{ca}$. del $1^{\mathrm{o}}$ Aprile la serie di uninodali cessa sul lago per un paio d'ore circa, dando luogo alla registrazione di lievi trinorlali, evidenti sopratutto a Sud. Quindi riprendono senza interruzione. I'ampiezza si fa particolarmente marcata nella notte dal 2 al Aprile, tanto da venire chiaramente registrate anche sulle sponde meridionali (fig. 18); la registrazione delle uninodali continua, come si è detto, fino all'interruzione delle misure, avvenuta la mattina del 4 Aprile 1961, alle $6^{\mathrm{h}} 45^{\mathrm{m}}$ circa.

III. - Confronti E Conclusioni. CaUse delle oscillazioni libere. Energia assoctata alle UNINOdali deI LAGIII DI Levico E DI Caldonazzo

1. - Malgrado qualche - del resto inevitabile, preventivata difficoltà, possiamo ritenere senz'altro soddisfacenti le due campagne di misure, condotte sui laghi di Levico e di Caldonazzo, e delle quali qui si è fatto ampio cenno. I risultati ottenuti hanno consentito di constatare la bontà dei dati forniti dall'applicazione di metodi, basati su diverse teorie idrodinamiche: la concordanza fra le risultanze della teoria e dell'osservazione va certo oltre ogni ottimistica previsione, spe- 


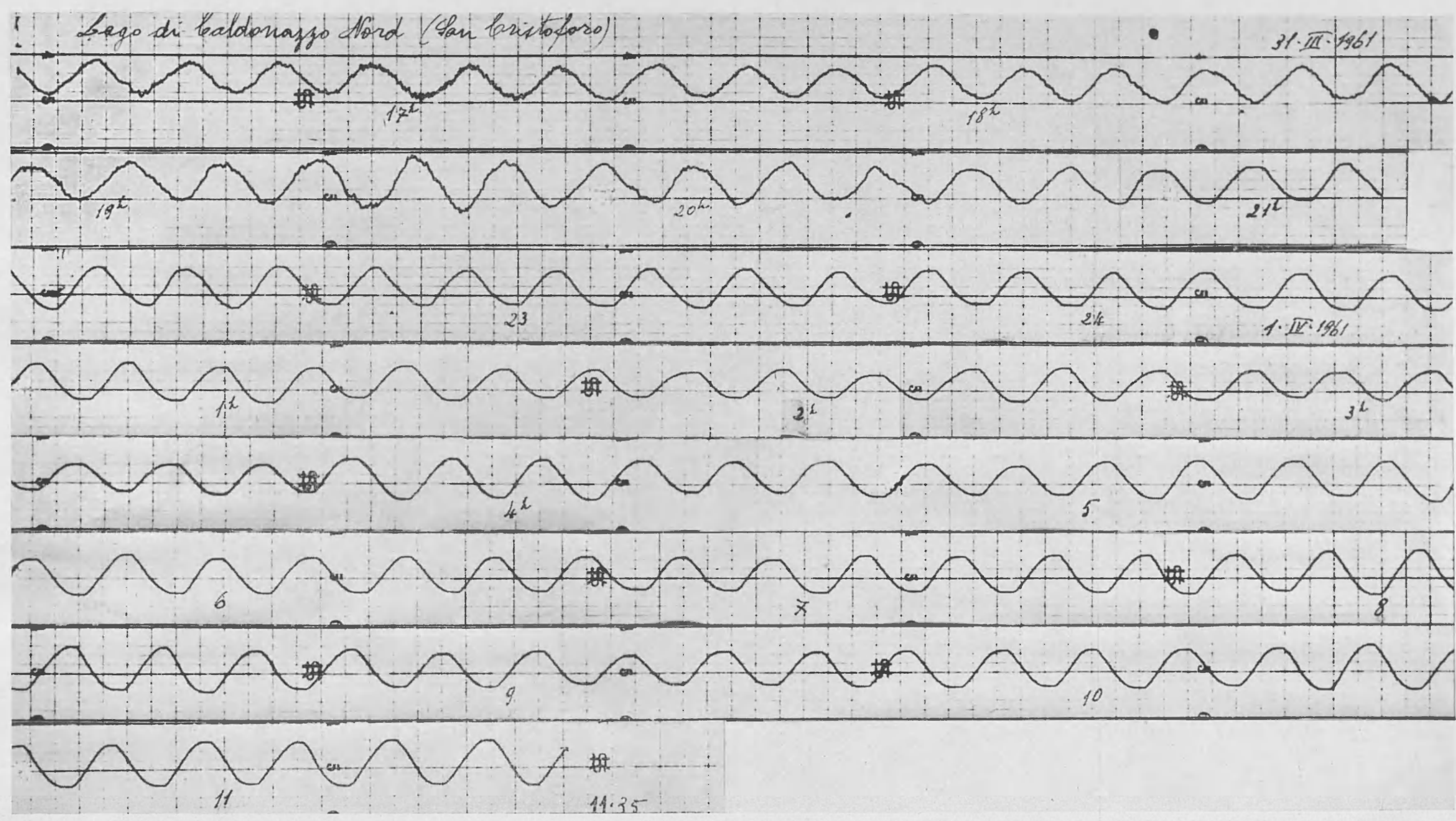

Fig. 17 - Lunga serie di uninodali a San Cristoforo. 
VALIDIT À DELLE TEORIE IDRODINAMICHE SULLE SESSE, ECC. 311

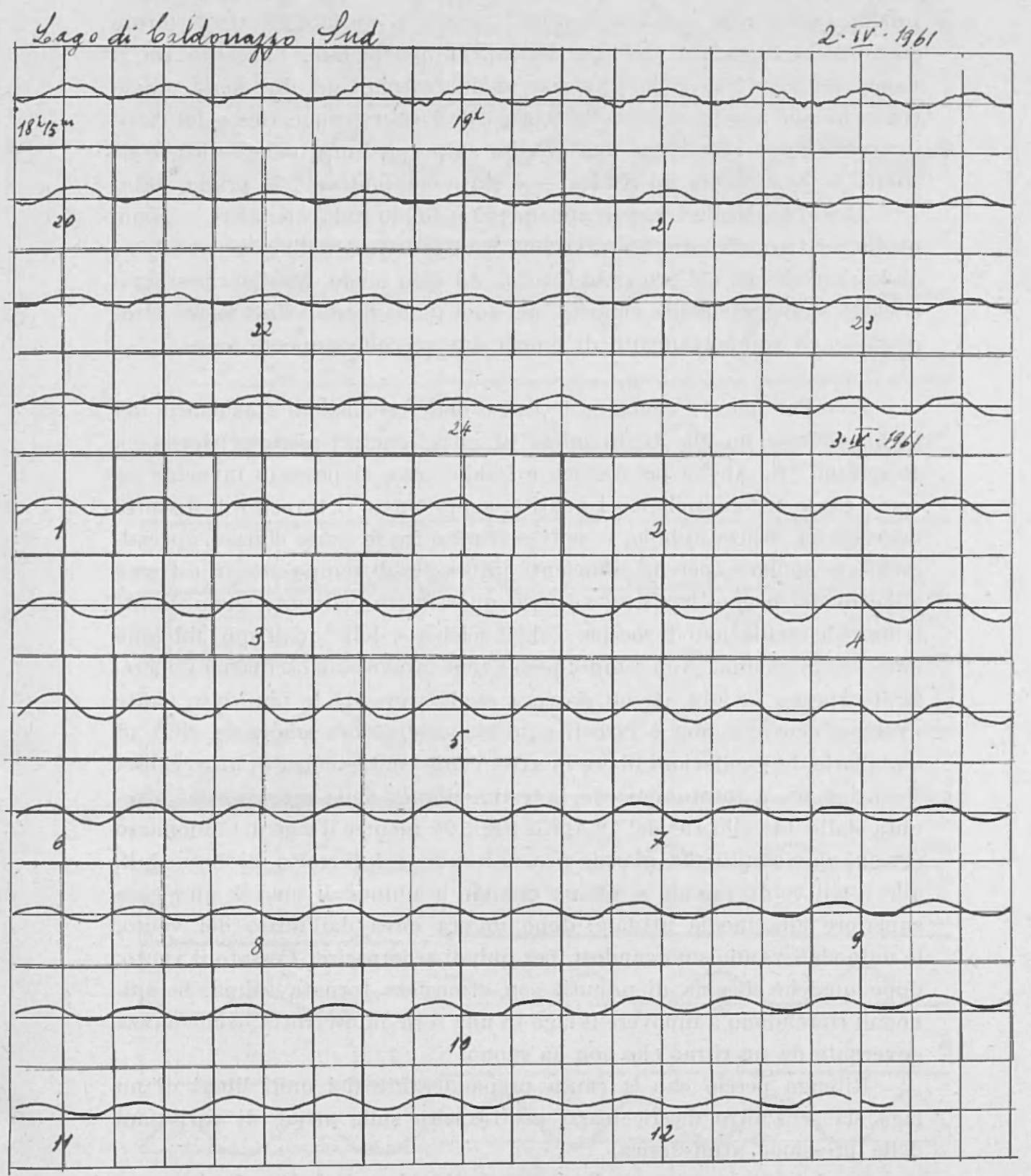

Fig. 18 - Attività normale di uninodali nella parte Sud del lago di Caldonazzo (7/10 dell'originale). 
cie se si tien conto della complessità dei problemi affrontati e delle approssimazioni rese necessarie dalla varietà e variabilità delle forme geometrico-geografiche dei due bacini. In particolare, l'accordo fra il valore del rapporto delle ampiezze ai due estremi dei due laghi, quale consegue dall'applicazione della teoria e dall'osservazione, riesce del tutto inatteso. E ciò costituisce una gradita sorpresa, tanto più che tale confronto - per quanto mi risulta — è stato compiuto per la prima volta.

Alcuni particolari vanno ancora più a fondo indagati; altri - come quello relativo alle correnti alternate, caratteristiche delle zone nodali andranno rilevati nel prossimo futuro. Ad ogni modo, fin d'ora possiamo ritenere sufficientemente chiarito, nei suoi fondamentali lineamenti idrodinamici, il comportamento di questi due piccoli, stupendi laghi.

2. - Per quanto concerne le cause delle oscillazioni stazionarie dei laghi, su esse ho già avuto modo di intrattenermi altrove (Garda $\left(^{6}\right)$, Bracciano ( $\left.{ }^{7}\right)$ ). Anche per Levico e Caldonazzo, si possono invocare come cause sistematiche - i venti e, sopra tutto, il transito di disturbi microbarici. Senza dubbio, i venti rientrano fra le cause efficaci, specialmente se soffiano, per un sufficiente intervallo di tempo, da un estremo all'altro del bacino lacustre; ad essi, quasi esclusivamente, sono da attribuire le oscillazioni di zoccolo "shelf-seiches ", delle quali qui abbiamo dato alcuni esempi. Non sempre però i venti provocano oscillazioni libere. Molto spesso, la loro azione provoca esclusivamente le più volte citate " shelf-seiches "; e non è raro il caso che addirittura smorzino, fino ad annullarle, le oscillazioni libere in atto, come conseguenza di altre cause. Vedasi, p. es., a questo riguardo, la testimonianza della registrazione ottenuta dalle $14^{\mathrm{h}}$ alle $19^{\mathrm{h}}$ del $1^{\circ}$ Aprile (fig. 19): mentre il lago di Caldonazzo era in movimento, da giorni, per una serie ininterrotta di uninodali, alle $14^{\mathrm{h}}$ il vento prende a soffiare quando le uninodali sono di ampiezza superiore alla media attuale: dopo un'ora circa dall'inizio del vento, le uninodali vanno smorzandosi, per quindi scomparire. Cessato il vento, dopo qualche diecina di minuti, con atmosfera tornata calma, le uninodali riprendono a muovere il lago in una serie ininterrotta, come danza governata da un ritmo che non ha suono.

Ritengo perciò che la causa preponderante dei moti liberi di un lago sia senz'altro da ricercarsi nel transito sulle acque di variazioni della pressione atmosferica.

Per intere giornate, con la superficie liquida calmissima, liscia come specchio, i limnografi registrano lunghissime teorie di oscillazioni libere. Solo nuclei di disturbi microbarici in transito possono essere invocati, 



M.

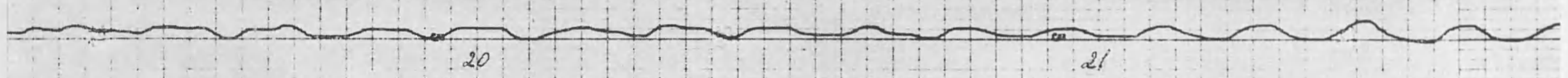

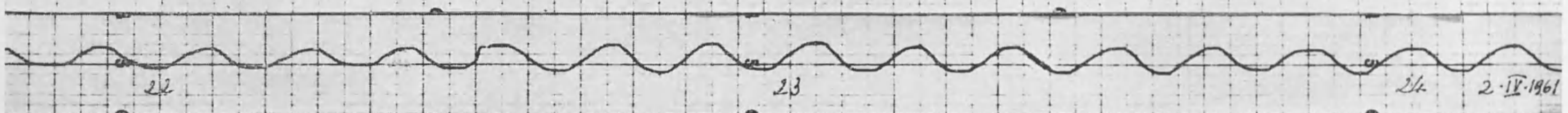

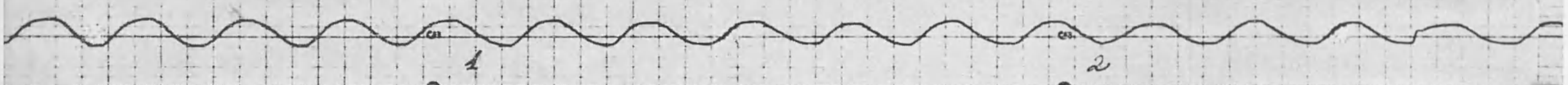



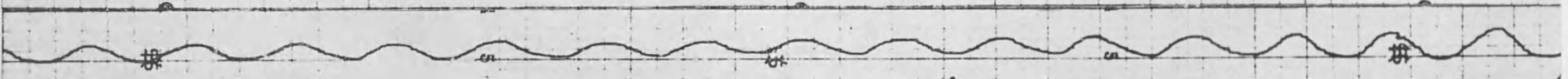

12 13

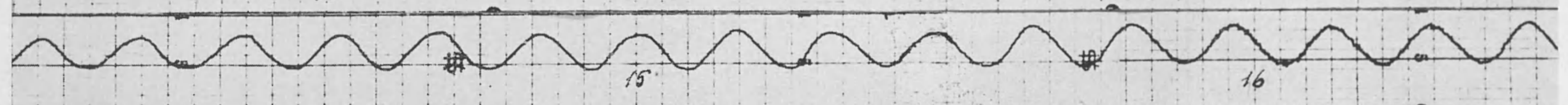

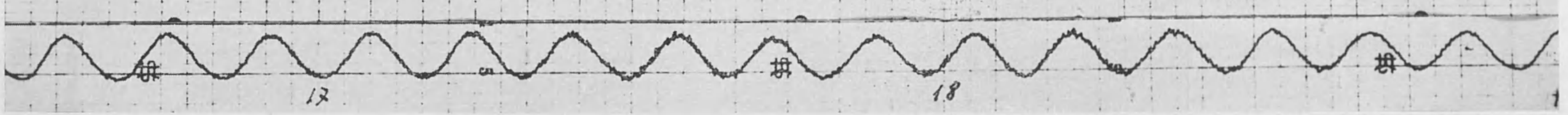


binodali. Verso le $20^{\text {h }}$ del $1^{\circ}$ Aprile si ha la ripresa dello uninodali. 
VALIDIT À DELLE TEORIE IDRODINAMICHE SULLE SESSE, ECC.

313

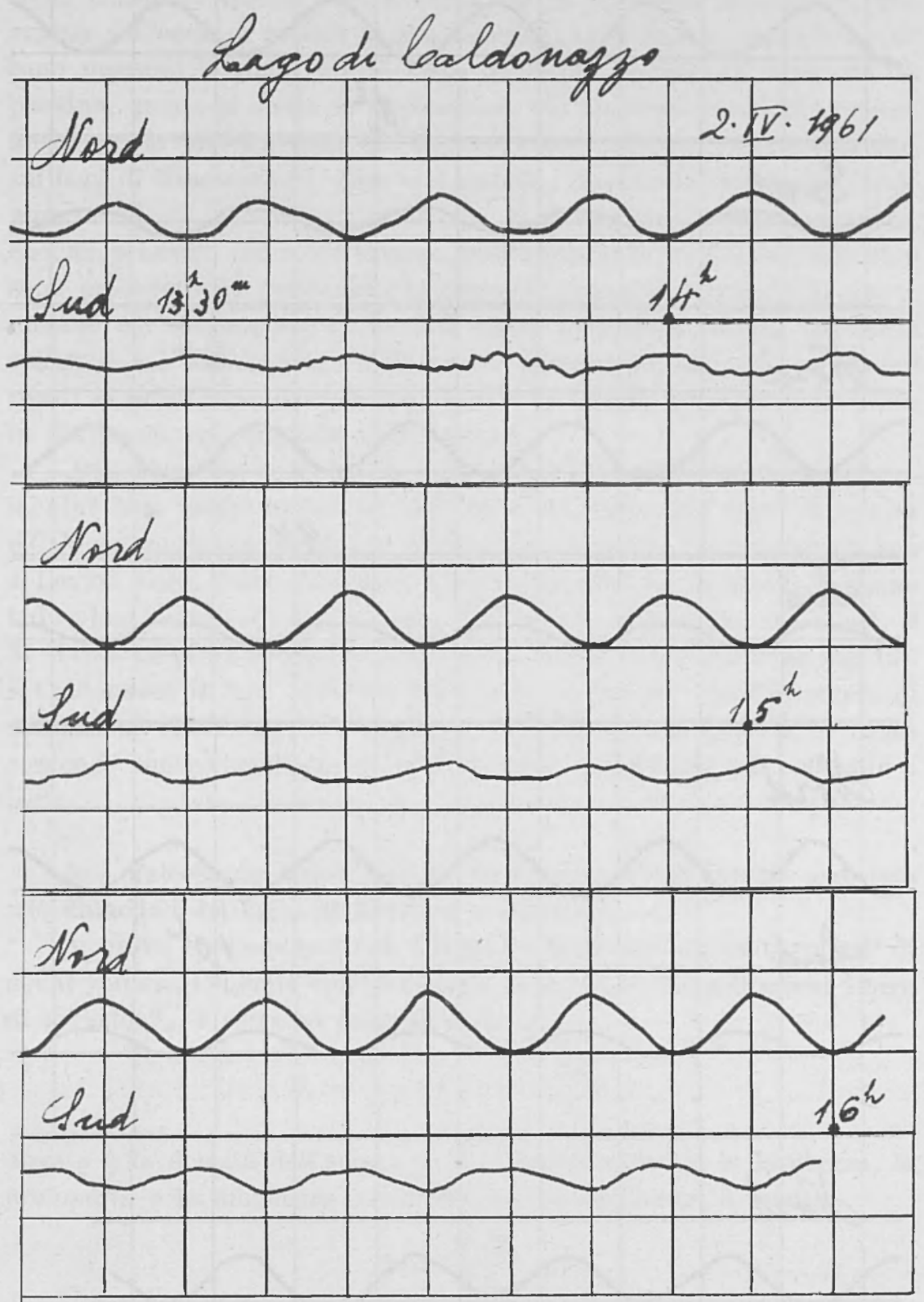

Fig. 20 - Contemporanea registrazione di uninodali sulle rive Nord e Sud del lago di Caldonazzo: conformemente alla teoria, a Nord si registra un'ampiezza circa 2,5 volte maggiore che a sud. 


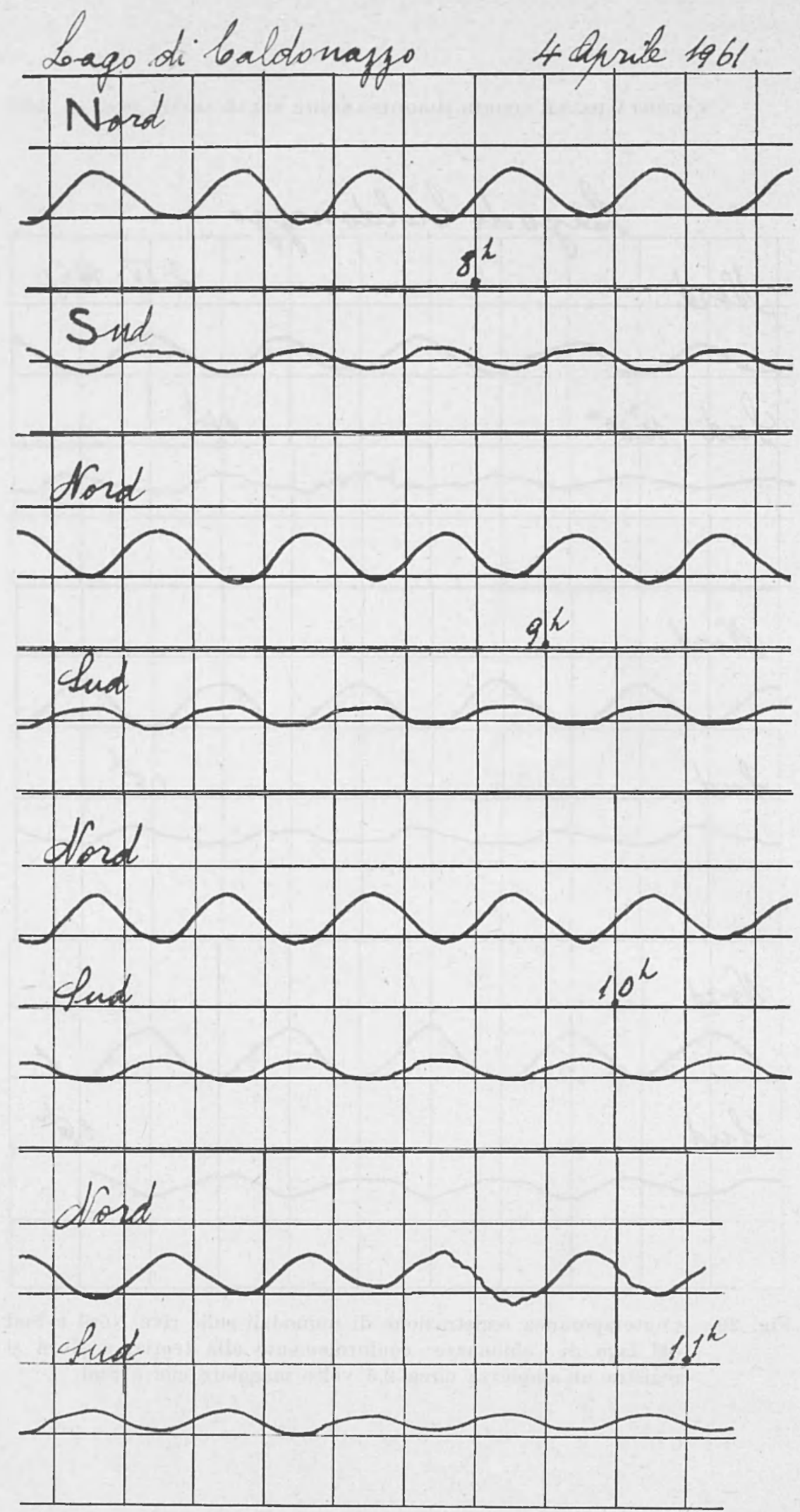

Fir. 21 - Contemporaneo alternarsi di uninodali al Lido di San Cristoforo e al Lido di Caldonazzo: il rapporto delle ampiezze è conforme alle indicazioni della teoria. 
come cause, in questi casi. I. noto che la pressione atmosferica può variare sull'onda di periodi o pseudo-periodi i più diversi; quando questi sono prossimi a quelli propri delle oscillazioni libere del lago, su cui passano, questo si mette in oscillazione. Ciò ho avuto modo di provare, anche con la testimonianza di contemporanee registrazioni microbariche, sul lago di Bracciano ( $\left.{ }^{7}\right)$. Non vi è dubbio, che questa conclusione vale pure per i laghi di Levico e Caldonazzo. ì sintomatico, a questo riguardo, che, sia a Levico che a Caldonazzo, molto spesso le oscillazioni uninodali sono precedute da oscillazioni multinodali (binodali e trinodali), che finiscono col sovrapporsi anche alle stesse uninodali: ritengo ciò possa spiegarsi col sopraggiungere di nuclei microbarici, animati su diversi ritmi: ad essi il lago risponde con i suoi ritmi silenti, come corda pizzicata in più punti, che diffonda suoni diversi.

Alle cause dei moti liberi, i due laghi rispondono contemporaneamente? Non necessariamente. Dal 30 al 31 Marzo, sui laghi di Levico e Caldonazzo hanno funzionato contemporaneamente due limnografi, uno a Levico Nord, l'altro al Lido di Caldonazzo. Ebbene, mentre il 30 Marzo i due laghi erano in oscillazione, ognuno con la propria uninodale, il 31 Marzo, mentre a Levico Nord si alternavano evidenti uninodali (fig. 12), a Caldonazzo il lago appariva calmo. Eे da ritenere che l'insorgere di oscillazioni libere nei due laghi sia contemporaneo, quando la causa contenga motivi multipli di perturbazione, adattabili ad entrambi $\mathrm{j}$ laghi.

3. - Calcoliamo infine l'ordine di grandezza dell'energia associata alle uninodali dei laghi di Levico e Caldonazzo.

Si prova che, equiparando i laghi in questione a parallelepipedi di ugual volume, l'energia cinetica media, relativa ad un'oscillazione libera di periodo $T_{o}$, è espressa dalla formula $\left(^{(8)}\right.$ :

$$
E_{c}=\frac{1}{4} \varrho b h l C^{2} \sin ^{2} \frac{2 \pi \tau}{I_{o}^{\prime}} t,
$$

dove $\varrho$ è la densità dell'acqua, $b, h, l$ rispettivamente la larghezza, la profondità e la lunghezza medie del bacino oscillante; è, inoltre,

$$
C-\frac{H \cdot 2 l}{T_{0} h}
$$

essendo $H$ l'ampiezza dell'oscillazione libera considerata.

Per il lago di Levico si ha:

$$
h=21,7 \mathrm{~m}, \quad l=2700 \mathrm{~m}, \quad T_{\mathrm{n}}=\hat{\mathrm{u}} .
$$


Per quanto concerne il valore di $b$, esso può essere dedotto dalla

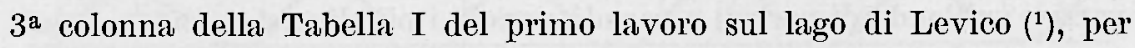
$h=21,7$. Ne consegue:

$$
b=346,9 \mathrm{~m} \text {. }
$$

Pertanto, fatto $\varrho=1 \mathrm{gr} / \mathrm{cm}^{3}$, nel caso di $H=3 \mathrm{~cm}$, consegue $C=2,07 \mathrm{~cm} / \mathrm{sec}$ e, quindi,

$$
\begin{aligned}
\dot{E}_{c} & =2,177 \cdot 10^{13} \mathrm{erg} \\
& =0,6 \mathrm{kwh} .
\end{aligned}
$$

Per $H=1 \mathrm{~cm}$ naturalmente l'energia si riduce in modo sensibile. Abbiamo infatti:

$$
C=0,69 \mathrm{~cm} / \mathrm{sec}
$$

e

$$
\begin{aligned}
E_{c} & =0,24 \cdot 10^{13} \mathrm{erg} \\
& =0,0666 \ldots \mathrm{kwh}
\end{aligned}
$$

Ad ogni modo, tenuto conto del fatto che l'ampiezza dell'uninodale nel lato settentrionale del lago è circa 3,5 volte quella osservata nel lato Sud, se ne conclude che l'energia associata all'uninodale è a Levico Sud circa $1 / 12$ di quella che si esplica a Nord.

Per il lago di Caldonazzo è

$$
h=\mathrm{m} 24,9, \quad 1=4100 \mathrm{n}, T_{\mathrm{o}}=10^{\mathrm{m}} .
$$

Dalla $3^{a}$ colonna della Tabella I del primo lavoro sul lago di Caldonazzo $\left({ }^{2}\right)$, si ha:

$$
b=1273 \mathrm{~m} \text {. }
$$

Per $H=2 \mathrm{~cm}$ è $C=1,1 \mathrm{~cm} / \mathrm{sec}$ e quindi (per $b=1270 \mathrm{~m}$ )

$$
\begin{aligned}
E_{c} & =3,9 \cdot 10^{13} \mathrm{erg} \\
& =1,08 \mathrm{kwh} .
\end{aligned}
$$

Anche in questo lago l'energia dell'uninodale è molto maggiore al Nord che non al Sud, dove risulta circa $1 / 7$ di quella che si manifesta a San Cristoforo.

Si tratta comunque di piccole energie, anche se nelle 24 ore esse non siano del tutto trascurabili. Nel lago di Levico infatti possono verificarsi, in un giorno, 240 uninodali, mentre in quello di Caldonazzo il loro numero puó essere di 144. 


\section{RIASSUNTO}

$S i$ riportano $i$ risultati delle registrazioni di oscillazioni libere e forzate, ottenute, in due diversi periodi, sui laghi di Levico e Caldonazzo. I valori ottenuti con il calcolo, $e$ discussi in precedenti lavori, vengono confermati con buonissima approssimazione; conformemente alla teoria, le ampiezze delle uninodali nei due laghi sono nettamente maggiori nella parte Nord dei laghi stessi; di quattro volte circa per il lago di Levico e di 2,5-3 circa per quello di Caldonazzo. Le differenze di livello riscontrate nei due laghi, nelle due diverse campagne, non influiscono sensibilmente sui valori dei periodi. Fra le cause delle oscillazioni stazionarie, predomina nettamente quella legata al transito di variazioni di pressione atmosferica. A volte il vento, anziché destare oscillazioni libere, pù̀ smorzare od annullare quelle già in atto.

Si determina il medio valore dell'energia, associata alle uninodali dei due laghi: essa è di $2.10^{13}$ erg per Levico Nord e $4.10^{13}$ erg per Caldonazzo Nord. Nella parte Sud dei laghi, l'energia viene notevolmente ridotta: di 1/12 circa per il lago di Levico e di 1/7 circa per quello di Caldonazzo.

\section{$S U M M A R Y$}

We report the results of the registrations of free and forced oscillations, which have been obtained during two different periods, on the lake of Levico and on the lake of Caldonazzo. The values obtained by calculation have already been discussed in previous works, and are now confirmed with a very good approximation; in accordance with the theory, the amplitude of the uninodals in both lakes are distinctly bigger in the northern part of these lakes; about four times in the Levico lake, and 2,5-3 times in the Caldonazzo lake. The level difference found in these two lakes, in the two different seasons, don't seriously infuence the values of the periods. Among the causes for stationary oscillations, the most important one is connected with the passage of variations of atmospheric pressure. At times the wind, instead of stirring free oscillations, would damp or even annul those already in progress.

We determine the average value of the energy associated to the uninodals of these two lakes: it is $2.10^{13}$ erg for the northern part of the Levico lake, and $4.10^{13}$ erg for the northern part of the Caldonazzo lake. In the southern part of both lakes the energy is considerably reduced: about 1/12 for the Levico lake, and about 1/7 for the Caldonazzo lake. 


\section{BIBLIOGRAFIA}

(1) Calor P., Oscillazioni libere del lago di Levico. "Ann. di Geofisica ", IV, 2, (1951).

( $\left.{ }^{2}\right)$ Caloi P., Spadea M. C., Sulle oscillazioni libere del lago di Caldonazzo, "Ann. di Geofisica ", V, 1 (1952)

$\left({ }^{3}\right)$ Calor P., Oscillazioni libere dei laghi di Caldonazzo e di Levico. "Ricerca Limn. del Centro di St. per la Geogr. Fis. del C.N.R. ", Bologna, (1952).

(4) Polli S., Le sesse dei laghi di Caldonazzo e di Levico, "Centro Studi per la Geogr. Fis. ", Bologna, (1952).

(5) Calor P., Sesse dell'Alto Adriatico con particolare riguardo al golfo di Trieste. "Rend. Comit. Talass. It., Mem. ", CCXLVII, (1938).

$\left(^{6}\right)$ Calor P., Spadea M. C., Le sesse del lago di Garda, Parte IV, "Ann. di Geofisica ", VI, 4, (1953).

(7) Caloi P., Peronaci F., Oscillazioni libere e forzate del lago di Bracciano. "Arch. di Oceanogr. e Limn. ", Venezia, (1958).

${ }^{8}$ ) Calor P., Oscillazioni libere del lago di Bracciano, loro caratteristiche e loro energia. "Ann. di Geofisica ", XI, 1, (1958). 